\title{
PERCEPÇÃO DE IDOSOS SOBRE O USO DE AASI CONCEDIDO PELO SISTEMA ÚNICO DE SAÚDE
}

\author{
Elderly perception on the use of hearing aids \\ given by the health system (SUS)
}

\author{
Indiara de Mesquita Fialho (1), Daniela Bortoli (2), Gabriella Gomes de Mendonça ${ }^{(3)}$, \\ Débora Frizzo Pagnosim ${ }^{(4)}$, Alessandro da Silva Scholze ${ }^{(5)}$
}

\begin{abstract}
RESUMO
Objetivo: conhecer a percepção de idosos sobre o uso de aparelhos de amplificação sonora individuais (AASI) concedidos pelo Sistema Único de Saúde (SUS). Métodos: estudo de caráter qualitativo. Foram realizadas entrevistas semi-estruturadas com 19 idosos, entre 60 e 86 anos, com adaptação binaural e tempo de uso de AASI entre seis meses e um ano. As entrevistas foram gravadas e transcritas para posterior leitura e análise temática. As categorias estabelecidas foram: dificuldades de comunicação sem AASI; o acesso ao AASI e o processo de adaptação ao uso; o impacto do uso do AASI na comunicação. Resultados: a dificuldade para se comunicar foi maior em situações em que há várias pessoas conversando; a dificuldade está em entender e não em ouvir, demonstrando uma preocupação em ressaltar o que sentem em ser surdo. A maioria dos participantes não realizou a adaptação do AASI anteriormente por não ter condições financeiras e revela que o que possibilitou o acesso foi a implantação do Serviço de Atenção à Saúde Auditiva. As dificuldades encontradas no processo de adaptação foram: mudança na percepção da própria voz, dificuldade de percepção de fala em ambiente ruidoso e no telefone. Relataram benefício com o uso de AASI na comunicação com familiares e com estranhos. Conclusão: destaca-se a importância do processo de acompanhamento para que as dificuldades de adaptação possam ser detectadas e minimizadas, para que efetivamente o sujeito obtenha benefício e satisfação com este tratamento.
\end{abstract}

DESCRITORES: Auxiliares de Audição; Idoso; Perda Auditiva; Percepção; Reabilitação

\section{INTRODUÇÃO}

Considerando que a diminuição da audição está relacionada ao envelhecimento, o aumento da

(1) Fonoaudióloga; Professora do Curso de Graduação em Fonoaudiologia e de Pós-Graduação em Audiologia da Universidade do Vale do Itajaí, UNIVALI, Itajaí, SC; Mestre em Saúde Pública pela Universidade de São Paulo.

(2) Aluna do Curso de Graduação em Fonoaudiologia da Universidade do Vale do Itajaí, UNIVALI, Itajaí, SC.

(3) Aluna do Curso de Graduação em Fonoaudiologia da Universidade do Vale do Itajaí, UNIVALI, Itajaí, SC.

(4) Fonoaudióloga; Docente do Curso de Graduação em Fonoaudiologia e de Pós-Graduação em Audiologia da Universidade do Vale do Itajaí, UNIVALI, Itajaí, SC; Mestre em Distúrbios da Comunicação Humana pela Universidade Federal de Santa Maria, convênio com a Universidade Federal de São Paulo - Escola Paulista de Medicina.

(5) Médico da Estratégia Saúde da Família do Município de Itajaí, SC; Docente do Curso de Graduação em Medicina da Universidade do Vale do Itajaí, UNIVALI, Itajaí, SC; Mestre em Saúde e Gestão do Trabalho.

Conflito de interesse: INEXISTENTE população idosa ocasiona um aumento no número de pessoas que apresentam algum tipo de problema auditivo.

A perda auditiva que caracteristicamente acomete os idosos é a presbiacusia, que acarreta uma redução na compreensão da fala, o que compromete o processo de comunicação e interfere no convívio social, na vida psicológica e profissional surgindo também sentimentos de insegurança, medo, depressão e isolamento ${ }^{1-5}$.

Sabe-se que a comunicação corresponde a uma necessidade essencial do ser humano; por meio dela a pessoa tem a possibilidade de adquirir novos conhecimentos e experiências, ela faz com que a pessoa continue ativa no seu convívio social e familiar, sendo assim, quando há uma redução desta capacidade logo se percebe a frustração diante da falta de compreensão e, em seguida, o isolamento e a depressão ${ }^{6}$.

Esta desvantagem, aliada ao estigma de "ser velho", favorece o isolamento social e familiar do 
idoso, pois o mesmo sente-se frustrado por não ouvir bem e não compreender bem o que os outros falam. Assumir a deficiência auditiva associa-se, na percepção dos idosos, a assumir que se está ficando "velho", e isso traz intrínseca a ideia de incapacidade ${ }^{7}$.

Como forma de minimizar a deficiência auditiva quando a mesma não é passível de tratamento medicamentoso ou cirúrgico, a conduta médica é a indicação do uso de aparelho de amplificação sonora individual (AASI) ${ }^{8}$.

Embora o conhecimento técnico-científico aponte que o uso do AASI possa auxiliar seus usuários na melhoria de sua qualidade de vida, no que se refere aos aspectos linguísticos, sociais e emocionais, sabe-se que muitos idosos não aceitam o uso deste dispositivo.

Este estudo vem ao encontro das diretrizes do Ministério da Saúde que ressalta a necessidade de um levantamento da situação do país em relação à deficiência auditiva; no que se refere ao número de portadores de problemas auditivos, os tipos de perda auditiva, o fornecimento da prótese e a satisfação do usuário após o recebimento, o que possibilitaria uma melhoria do planejamento das ações em Atenção à Saúde Auditiva ${ }^{9}$.

Apesar do avanço tecnológico dos sistemas acústicos modernos, a satisfação do usuário continua sendo um desafio para os audiologistas, e as altas taxas de abandono do uso do AASI, um problema para os serviços de saúde. Desta forma, monitorar a satisfação é importante para avaliar os procedimentos clínicos e garantir os propósitos de qualidade dos serviços e por que a satisfação reflete a realidade dos resultados de saúde ${ }^{10}$.

Assim, considera-se que, conhecer a percepção do uso do AASI em idosos poderá auxiliar os acadêmicos e profissionais da área a compreenderem os sentimentos e necessidades destes participantes, o que pode refletir na qualidade e humanização do atendimento. A avaliação da satisfação com o uso de AASI tem importância uma vez que verifica os resultados da adaptação a partir do ponto de vista do usuário ${ }^{11}$. Somente o usuário de próteses auditivas poderá deliberar quais as dificuldades encontradas com o uso sistemático da amplificação, no transcorrer da reabilitação ${ }^{9}$.

No processo de seleção e adaptação de AASI as informações obtidas por avaliações subjetivas podem ser mais significativas do que as obtidas por meio de avaliações objetivas ${ }^{12}$.

Além disso, conhecer a percepção dos usuários do AASI é também uma forma de avaliar os resultados do serviço prestado a esses indivíduos.

Com base nesses pressupostos, este trabalho teve como objetivo conhecer a percepção de idosos sobre uso de AASI concedido pelo Sistema Único de Saúde (SUS).

\section{MÉTODOS}

Este estudo, de caráter qualitativo, foi realizado com indivíduos que receberam AASI no ano de 2006, em um Serviço credenciado ao SUS como de média complexidade. O Serviço presta atendimento a 53 municípios da região do Vale, Médio e Alto Vale do Itajaí-SC.

Participaram desta pesquisa 19 idosos na faixa etária de 60 a 86 anos, sendo 10 do sexo masculino e nove do sexo feminino, todos com adaptação binaural, com perda auditiva bilateral de grau moderado a severo, do tipo misto ou neurossensorial. $\mathrm{O}$ tempo de uso de AASI variou de seis meses a um ano, sendo que os entrevistados não haviam feito uso de AASI anteriormente.

O número de participantes foi determinado no decorrer da coleta de dados, a partir do momento em que houve a "reincidência" das informações. A amostra foi constituída de maneira diversificada no que se refere ao sexo, ocupação, cidade em que reside, tipo e grau de perda auditiva, a fim de possibilitar a apreensão de semelhanças e diferenças ${ }^{13}$.

A coleta de dados se deu por meio de entrevista semi-estruturada com roteiro que visou abordar os seguintes temas: verificar como os idosos percebem sua comunicação sem o uso de AASI; verificar como os idosos percebem sua comunicação com o uso de AASI; identificar os fatores que influenciam o uso de AASI.

As entrevistas foram realizadas mediante aceite dos participantes e com o conhecimento e assinatura do termo de consentimento livre e esclarecido, sendo gravadas em gravador digital. As mesmas foram realizadas em uma sala fora do ambiente do serviço de atenção à saúde auditiva e, no momento da realização, os indivíduos faziam uso de seus aparelhos auditivos.

A análise temática dos dados foi realizada seguindo o caminho proposto por Minayo ${ }^{13}$. As categorias de análise estabelecidas foram: dificuldades de comunicação sem AASI - o que causa em mim e o que causa no outro; dificuldades no acesso e na adaptação de AASI; o impacto do uso do AASI na comunicação.

Os resultados são apresentados por meio de transcrição literal da fala dos participantes, destacando-se os principais trechos em itálico. Os participantes são identificados por meio da letra $S$, seguida de um número de um a 19.

Salienta-se que esta pesquisa foi realizada mediante a aprovação do Comitê de Ética em Pesquisa da UNIVALI sob parecer no 539/2006. 


\section{RESULTADOS}

\section{Categoria 1 - Dificuldades de comunicação sem AASI - o que causa em mim e o que causa no outro}

Ao relatarem as dificuldades de comunicação sem o uso de AASI, observa-se que a dificuldade é maior em situações em que há várias pessoas conversando, os participantes afirmam que sua dificuldade está em entender e não em ouvir. Além disso, há uma preocupação em distinguir o que sentem do ser surdo.

$S$ 1: A pessoa surda, conversando não ouve bem, por exemplo, não é que seja surda. Na roda de amigo todo mundo conversando, fala uma coisa e outra e a gente não entende. $O$ surdo, por causa da surdez, da velhice, a gente escuta os sons, só que a gente não entende as palavras, esse que é o problema.

Na percepção dos participantes, a dificuldade para ouvir também causa um "incômodo" para os familiares. Uma das queixas encontradas foi em relação ao volume da televisão, pois a intensidade alta "irrita" os mesmos.

S 4: Não escutar incomoda, porque a televisão tinha que ser alta e os filhos reclamavam.

Em relação a si próprios, os participantes relatam que o maior incômodo causado é não conseguir acompanhar a celebração da missa e não entender o que os amigos falam durante a conversação. Além disso, os relatos registram a dificuldade para entender a fala de pessoas desconhecidas, como ocorre nos postos de saúde. falava.

$S$ 4: Pra ir na igreja não escutava nada que

S 11: Eu ia à missa, o que o Padre falava lá, muitas coisas eu não escuto.

$S$ 15: Precisa ficar repetindo as coisas e cada vez foi piorando. Agora então, Nossa! Tava difícil! No relacionamento com meus familiares, eu tinha que gritar ou então ficar repetindo. Tudo era sofrido, pra sair e conversar com as pessoas em estabelecimento público, assim tipo posto (referindo-se ao posto de saúde).

\section{Categoria 2 - Dificuldades no acesso e na adaptação de AASI}

A maioria dos participantes não fez uso de AASI anteriormente, por não ter condições financeiras de comprar um aparelho auditivo. Alguns relatam ter adiado a procura pelo aparelho, pois na idade que estão "não vale a pena fazer mais sacrifícios" ou "quando o aparelho chegar talvez eu nem esteja mais aqui".

S 12: Eu descobri uma clínica que fazia tudo, aí eu fui lá pra fazer os exames. A guria pôs o apareIho no meu ouvido, aquele troço, tão ruim. Eu disse: não, não quero. Aí, não fiz mais nada. Aí pensei, vou pagar um aparelho caro e não usar? Esse sacrifício eu não vou fazer. E elas sempre brigando comigo, as minhas netas: Faz vó, faz, faz, faz. Daí essa possibilidade de vir aqui. Daí onde eu fiz, onde eu entrei em contato com os médicos fiz a ficha lá no Posto Central e aguardei a chamada. Me chamaram e eu vim.

Nas falas a seguir, os participantes relatam o caminho percorrido na rede de saúde para obter o AASI e registram que todo o processo, desde o encaminhamento para a secretaria de saúde, levou em torno de um ano. A palavra "doando" aparece na fala do $S 15$ denotando a percepção de que este benefício Ihe é concedido como "favor" e não como "direito".

$S$ 15: A gente ficou sabendo que a Secretaria tava doando aparelho. Daí a gente veio aqui se informar. Aí marcaram a data, a gente teria que vir encaminhada pela Secretaria de Saúde. Aí foi marcado com o otorrino. Eu fui lá, consultei tudo e encaminhei os xerox dos papéis. Daí ela mando pra cá e avisaram a gente quando foi pra vir. Daí, á foi tirado o molde do aparelho tudo e em tempo, em prazo de um ano e pouquinho eu consegui tudo.

Evidenciou-se também a dificuldade em aceitar o uso de AASI, em função da questão estética. $S$ 12: "Eu tenho uma outra companheira, amiga minha, ela pôs o aparelho, mas ela fez particular, ela põe só o aparelho dentro do ouvido não tem assim, como esse ai" (referindo-se ao modelo de AASI, retroauricular e intracanal). Esta fala também evidencia a percepção de que no serviço particular o indivíduo pode obter aparelhos de melhor qualidade.

Os participantes apontam algumas dificuldades em relação ao processo de adaptação ao uso do AASI. Relatam situações de escuta nas quais não observam benefício com o uso do aparelho auditivo e dificuldades no manuseio do AASI.

Escutar a fala no telefone com o uso de AASI foi uma das dificuldades de escuta relatada.

$S$ 18: Mas ainda não me acostumei bem de atender ao telefone com o aparelho parece que não funciona normal. Parece que não peguei bem o jeito ainda. 
Alguns relatam que a sua voz mudou com o uso do aparelho auditivo e isso causa incômodo. Esta queixa pode ter relação com o efeito de oclusão causado com a inserção do molde.

$S$ 18: Custa um pouco até a gente se acostuma, né? Faz barulho, a voz da gente fica diferente.

No que se refere ao ruído, os relatos apontam que o mesmo é uma das maiores dificuldades encontradas pelos participantes em relação ao uso do aparelho auditivo, uma vez que a presença de ruído gera desconforto. As principais situações relatadas foram: o som dos bailes, dos carros e de aglomeração de pessoas.

$S$ 4: Quando você coloca, você escuta até o pneu no carro lá na rua. Então eu fazia assim, durante o dia eu não colocava muito, eu colocava mais à noite quando tava vendo televisão, assim eu fiz uma semana, fui colocando aos poucos, né? Até que aquele barulho irritante da moto, que eu moro bem numa esquina assim, até que o barulho irritante da moto, dos pneus dos carros foi se acalmando, daí logo me acostumei. (...) é difícil quando a gente não escuta.

Outra dificuldade relatada foi em relação à inserção e retirada do AASI, devido a alterações motoras. Isso fica evidente quando o S10 relata sua dificuldade para manipulação do AASI "eu tenho um defeito físico" ou quando o S14 refere "Me deu derrame e essa mão é boba".

Esses relatos apontam para a importância de orientar e esclarecer as dúvidas no momento da entrega do aparelho e nos retornos para o acompanhamento.

\section{Categoria 3 - 0 benefício do uso do AASI na comunicação}

Os relatos apontam o benefício do uso do aparelho auditivo em diferentes situações de comunicação.

$S$ 4: Ah, eu acho que foi muito bom porque levantou a estima, né? A gente se sente melhor, porque escuta as pessoas, parece que convive mais, né? Porque antes ficava mais retraída, porque não escutava os papos. Pra escutá tinha que tá bem pertinho, senão não escutava nada, se eu saía dois metro já não escutava nada. Então, eu acho que foi muito bom.

S 11: Tá tudo maravilhoso. Eu não posso me queixar. É um companheiro (referindo-se ao AASI) que eu arrumei pro resto da minha vida. É hoje em dia (...) melhorou tudo pra mim, até a minha voz.
$S$ 15: $A$ gente não escutava agora escuta, a comunicação com as pessoas, com a minha filha, com quem quer seja mudou completamente. Tá diferente (...) Acho que a minha vida mudou muito, pra melhor. A comunicação em casa e com o pessoal assim de fora também, quando a gente sai, mudou totalmente.

As falas registram o benefício trazido pelo uso de AASI em situações de conversas com familiares e com outras pessoas. O benefício é percebido quando participam em reuniões da comunidade, incluindo grupos de terceira idade, nas celebrações religiosas, em consultas médicas e para assistir televisão.

S 1: Agora entende melhor o som, (...) ir a uma reunião, por exemplo, aqui no parque Dom Bosco tem a reunião, agora eu tenho condição de ir. Porque antes eu ia em reunião, mas não escutava.

S 4: É muito bom, a gente vai por aí conversa, escuta a conversa dos outros. Primeiro eu não escutava nada, tinha que ficar quietinha, então ficava na minha, porque não escutava. Agora não, agora participo das conversa, porque a gente se reúne muito com a terceira idade, passeia muito, sabe?

O S18 relata a mudança que percebeu após iniciar o uso de AASI, destaca-se nesta fala a autonomia adquirida para ir às consultas médicas, o que antes não era possível, em função da dificuldade auditiva.

S 18: Mudou pra vê televisão e na igreja ou quando eu vou na médica. Uma época sempre alguém ia comigo, mas agora todo mundo trabalha, então vô sozinha.

\section{DISCUSSÃO}

Este estudo possibilitou registrar a percepção da perda auditiva e do uso do AASI por idosos. Observou-se que a perda auditiva causa, no idoso, dificuldades de comunicação que acarretam, em alguns casos, isolamento da família e da sociedade.

As dificuldades de audição e de compreensão durante a comunicação podem ocasionar interrupção das interações sociais, pois a associação da velhice com a dificuldade de comunicação pode alterar e intervir negativamente na qualidade de vida do idoso ${ }^{14}$. A dificuldade é maior em situações em que há várias pessoas conversando, sendo que os participantes afirmam que sua dificuldade está em entender e não em ouvir e demonstram uma preocupação em distinguir o que sentem do ser surdo. O fato de escutar, mas não entender, causa 
um "estranhamento" e uma dificuldade em compreender o que está ocorrendo, podendo se confundir com o fato de estar envelhecendo e/ou com a possibilidade de estar "perdendo o juízo" 7.

Em outro estudo realizado com idosos socialmente ativos, com queixa de dificuldade auditiva, $75 \%$ dos entrevistados relataram como queixa principal a dificuldade para conversar em ambiente ruidoso ${ }^{15}$. Esta mesma queixa foi referida pelos usuários de próteses auditivas em um estudo realizado no estado do Paraná, sendo fundamental o processo de adaptação para garantir o uso efetivo das mesmas nesses ambientes ${ }^{16}$.

Em relação aos familiares, a percepção dos participantes é que a dificuldade para ouvir é um "incômodo", em função do volume alto da televisão ou da necessidade de repetir o que foi falado, há uma preocupação em relação à segurança ao andar na rua, pelo fato de não ouvir o ruído dos carros, correndo o risco de atropelamento.

Nesse sentido, é imprescindível à implantação de um programa de reabilitação, para que o idoso e seus familiares encararem os obstáculos advindos da perda auditiva, o que pode gerar uma boa adaptação ao uso do AASI e uma facilitação na reinserção dos participantes idosos junto ao convívio social da família, dos amigos e dos companheiros de trabalho ${ }^{17}$.

Destaca-se que o uso do AASI é essencial para manter a saúde física e mental do idoso, pois possibilita ao mesmo uma melhora na participação na comunidade em que está inserido, também no convívio familiar, melhorando, assim, sua qualidade de vida ${ }^{18}$.

Em relação ao acesso ao uso de AASI, as falas revelam que o que possibilitou o acesso foi a implantação do Serviço de Atenção à Saúde Auditiva. Os participantes citam a Secretaria de Saúde, o Governo Federal, o médico e a instituição onde o serviço está localizado como via de acesso a este recurso. Verificou-se ainda, a crença de que no serviço particular teriam a possibilidade de receber um AASI de melhor qualidade do que o concedido por meio do SUS, isto foi observado quando um dos indivíduos refere-se ao tipo de AASI, retroauricular ou intracanal. No entanto, a Portaria 589 da secretaria de Atenção à Saúde, prevê a concessão de aparelhos de ambos os tipos, deste modo os indivíduos têm a possibilidade de receber estes apareIhos por meio do SUS ${ }^{19}$.

Em pesquisa realizada em uma instituição pública no estado do Tocantins foi encontrado alto índice de insatisfação em relação à imagem pessoal com o uso de AASI retroauricular ${ }^{20}$. Cabe então ao profissional avaliar junto com o candidato ao uso de AASI qual a melhor opção para cada caso, levando em consideração os diversos fatores envolvidos nesta escolha, entre eles: a aceitação da perda auditiva, a motivação para obter ajuda para minimizar as necessidades de comunicação, as preocupações financeiras, o nível intelectual e social do deficiente auditivo, além das necessidades estéticas e acústicas ${ }^{21}$. Para tanto, o profissional deve estar aberto para "ouvir" o indivíduo e estabelecer uma relação de confiança e deste modo abrir caminho para a orientação e aconselhamento efetivos.

No processo de adaptação ao AASI os participantes relataram as seguintes dificuldades: escutar a fala no telefone, adaptar-se à mudança na percepção da própria voz, compreender a fala em ambiente ruidoso, inserir e remover o AASI. Destaca-se que a principal dificuldade relatada é ouvir em ambiente ruidoso.

Em outro estudo, realizado em um Serviço de Atenção a Saúde Auditiva no Estado do Ceará, a principal queixa referida pelos usuários de AASI, também foi em relação ao uso deste dispositivo em ambientes ruidosos ${ }^{22}$. O progresso ocorrido até o momento tem auxiliado muito o deficiente auditivo em um aperfeiçoamento da audição para a fala, principalmente quando este se encontra em ambiente silencioso. Contudo, o desempenho que o sujeito apresenta, em ocasiões com ruído competitivo, ainda não alcançou um nível de melhora satisfatório ${ }^{23}$.

Essas dificuldades de adaptação ao uso de AASI podem se caracterizar como uma fase do processo de adaptação que envolve vários fatores: de ordem social, cultural, emocional, de questões técnicas de seleção das características do AASI ou como reflexo de uma dificuldade de percepção auditiva. A dificuldade de manipulação e ajuste do aparelho pode estar relacionada à redução progressiva da capacidade visual e das habilidades motoras constantemente encontradas nos idosos ${ }^{24}$.

Em outro estudo realizado com usuários atendidos em uma instituição pública no Estado do Rio Grande do Sul, os indivíduos entrevistados relataram dificuldades relacionadas aos moldes ou cápsulas auriculares, em relação às queixas e/ou problemas relacionados ao funcionamento das próteses auditivas as pesquisadoras encontraram: intensidade forte; intensidade fraca, desconforto, na maioria das vezes, relacionados ao uso binaural; dores de cabeça com frequência; dificuldades no ruído; ouvir, mas não entender; não ouvir de longe ${ }^{9}$.

Em todos os casos o acompanhamento é imprescindível, o profissional pode, junto com o sujeito, compreender as suas dificuldades e buscar soluções. A orientação e o acompanhamento são a chave do sucesso do uso efetivo do aparelho auditivo pelo idoso, não devendo, portanto, ser minimizada a sua importância no processo de adaptação de aparelhos para estes participantes 9,24,25. 
Apesar das dificuldades, os participantes entrevistados percebem o benefício do uso de AASI para a sua comunicação. Benefício observado nas seguintes situações: em conversas com familiares e com estranhos, em reuniões da comunidade, na igreja, em consultas médicas, para assistir televisão e em grupos de terceira idade. As falas revelam que o uso do AASI diminui o isolamento dos idosos e aumenta a autonomia nas atividades que envolvem o contato com o outro, uma vez que se sentem mais seguros em situações de comunicação. Uma pesquisa com a mesma população deste estudo constatou que $83,87 \%$ dos idosos entrevistados relataram melhora na habilidade para ouvir em diferentes situações ${ }^{26}$. Em uma pesquisa sobre o sentido da deficiência e o uso de aparelho auditivo para idosos, as autoras observaram que os aparelhos auditivos melhoraram o desempenho nas situações de comunicação de todos os idosos entrevistados, com repercussão positiva nas relações sociais, apesar de ainda relatarem dificuldades em algumas situações ${ }^{27}$.

A adaptação de próteses auditivas não é apenas fundamentada nas medidas objetivas que avaliam a audição. Atualmente, considera-se muito a avaliação do paciente em relação ao desempenho do aparelho, sua aceitação, benefício e satisfação ${ }^{28}$. A satisfação é influenciada pelo benefício percebido, mas abrange outros aspectos como: as expectativas do usuário, os custos financeiros e psicológicos envolvidos, os problemas encontrados no percurso de reabilitação e as dificuldades de comunicação que ainda permanecem mesmo com o uso da amplificação ${ }^{11}$.

\section{CONCLUSÃO}

Os dados trouxeram reflexões relacionadas à importância do processo de acompanhamento para que as dificuldades de adaptação possam ser detectadas e minimizadas, cabendo ao fonoaudiólogo trabalhar com as questões do estigma e da aceitação da perda auditiva, além das questões técnicas, para que efetivamente o sujeito obtenha benefício e satisfação com esse tratamento. No entanto, o profissional deve estar atento ao fato de que nem sempre as informações por ele repassadas são compreendidas ou aceitas pelos participantes.

Além disso, os dados apontam para a necessidade de orientar a população em geral e os profissionais de saúde em relação às dificuldades de comunicação dos idosos e sobre a necessidade do uso de estratégias de comunicação, a fim de minimizar os entraves da comunicação gerados pela deficiência auditiva.

\begin{abstract}
Purpose: to know elderly perception of hearing aids use given by the health system (SUS). Methods: qualitative study. Semi-structured interviews were carried out with 19 subjects with age ranging from 60 to 86 years, with binaural adaptation and using hearing aids from six months to one year. The interviews were recorded and transcribed for further reading and thematic analysis. The settled categories were: Difficulties of communication without hearing aids; The Access to the hearing aids and The Adaptation Process; The impact of hearing aids use in communication. Results: communication difficulty is higher in situations where there are many people talking; the difficulty is not in listening but in understanding; they show concern about distinguishing what they feel being deaf. Most subjects did not have previous hearing aids adaptation due to financial difficulties and revealed that their access to hearing aids was through implementation of Hearing Health Care Service. The difficulties concerning the adaptation process were: changing the perception of their own voice, difficulty in understanding the communication in noisy environment and on the phone. They reported the benefits of hearing aids use in communication with relatives and strangers. Conclusion: the monitoring process importance is highlighted so that the difficulties for adjustment can be effectively detected and minimized. Such being the case, the patient gets the benefit and satisfaction from the treatment.
\end{abstract}

KEYWORDS: Hearing Aid; Aged; Hearing Loss; Perception; Rehabilitation

\section{REFERÊNCIAS}

1. Russo ICP. Distúrbios da audição: a presbiacusia. In: Russo CP. Intervenção fonoaudiológica na terceira idade. Rio de Janeiro: Revinter; 1999. p. 57-82.

2. Magni C, Freiberger F, Tonn K. Avaliação do grau de satisfação entre os usuários de amplificação 
de tecnologia analógica e digital. Rev Bras Otorrinolaringol. 2005; 71(5):650-7.

3. Carvalho CRS, Reis AS, Jacobina GS, Silva VCM. Maturidade e envelhecimento: um enfoque fonoaudiológico. Fono Atual. 2003; 24(6):4-9.

4. Magni C, Armentano JN, Moreira PS, Winter EW. Investigação do grau de satisfação entre usuários de amplificação monoaural e binaural. Dist Comun. 2005; 17(3):323-32.

5. Pinzan-Faria VM, lório MCM. Sensibilidade auditiva e autopercepção do handicap: um estudo em idosos. Rev Dist Comun. 2004; 16(3):289-99.

6. Ferreira MIDC, Signor RC. O perfil do idoso usuário de prótese auditiva: um estudo da satisfação. Rev Fonoaudiol Bras. 2006; 4(2):1-2.

7. Fialho IM. Perda auditiva em idosos na percepção dos participantes [dissertação]. São Paulo (SP): Universidade de São Paulo; 2001.

8. Dan IB, lório MCM. Dificuldade e desvantagem auditiva: estudo em idosos na adaptação de próteses auditivas. Fono Atual. 2004; 29(7):50-5.

9. Freitas CD, Costa MJ. Processo de adaptação de próteses auditivas em usuários atendidos em uma instituição pública federal - parte I: resultados e implicações com o uso da amplificação. Rev Bras Otorrinolaringol. 2007; 73(6):744-51.

10. Veiga LR, Merlo ARC, Mengue SS. Satisfação com a prótese auditiva na vida diária em usuários do Sistema de Saúde do Exército. Rev Bras Otorrinolaringol. 2005; 71(1):67-73.

11. Almeida K. Avaliação dos resultados da intervenção. In: Almeida K, lório MCM. Próteses auditivas: fundamentos teóricos e aplicações clínicas. 2. ed. São Paulo: Lovise; 2003. p. 335-55. 12. Assayag FHM, Russo ICP. Avaliação subjetiva do beneficio e dos efeitos proporcionados pelo uso de amplificação sonora em indivíduos idosos. Dist Comun. 2006; 18(3):383-90.

13. Minayo MCS. O desafio do conhecimento: pesquisa qualitativa em saúde. 4. ed. São Paulo: Hucitec-Abrasco; 1999. 269p.

14. Gamburgo LJL, Monteiro MIB, Chun RYS. Questões sobre a atenção à saúde no enveIhecimento no âmbito da fonoaudiologia. Dist Comun. 2006; 18(1):111-7.

15. Lautenschlager L, Tochetto T, Gonçalves MS, Barreto DCM, Moro MP, Bolzan GP. Percepção de

RECEBIDO EM: 17/11/2008

ACEITO EM: 12/03/2009

Endereço para correspondência:

Indiara de Mesquita Fialho

Rua Amazonas, 347

Balneário Camboriú - SC

CEP: 88339-075

E-mail: indiaram@terra.com.br idosos socialmente ativos quanto às desvantagens causadas pela dificuldade auditiva. Einstein. 2008; 6(2):155-8.

16. Rosa MRB, Dante G, Ribas A. Programa de orientação a usuários de prótese auditiva e questionários de auto-avaliação: importantes instrumentos para uma adaptação auditiva efetiva. Arq Int Otorrinolaringol. 2006; 10(3):220-7.

17. Guarinello AC, Cruz MCM. O perfil dos idosos protetizados na clínica de fonoaudiologia da Universidade Tuiti do Paraná. Fono Atual. 2006; 8(35):59-64.

18. Ribas A, Rosa M, Dante G, Martins-Basseto J. SOS prótese auditiva: relato de um estudo de caso realizado na Clínica de Fonoaudiologia da UTP. Tuiuti: Ciência e Cultura. 2006; 38:9-18.

19. Brasil. Ministério da Saúde, Portaria no 589, 2004. Disponível em: URL: http://portal.saude.gov.br/ portal/sas/sapd/visualizar_texto.cfm?idtxt=22663.

20. Carvalho JSA. Satisfação de idosos com aparelhos auditivos concedidos no estado do Tocantins. Arq Int Otorrinolaringol. 2007; 11(4): 416-26.

21. Campos CAH, Russo ICP, Almeida K. Indicação, seleção eadaptação de próteses auditivas: princípios gerais. In: Almeida K, lório MCM. Próteses auditivas: fundamentos teóricos e aplicações clínicas. 2. ed. São Paulo: Lovise; 2003. p. 35-54.

22. Batista ACM, Sampaio FM. Nível de satisfação dos idosos usuários de próteses auditivas doadas pela APAC-NAMI-UNIFOR. Rev Bras Promoção da Saúde. 2005; 18(1):7-10.

23. Costa LP, lório MCM. Próteses auditivas: avaliações objetivas e subjetivas em usuários de amplificação linear e não-linear. Pró-Fono. 2006; 18(1):21-30.

24. Russo ICP, Almeida K, Freire GMF. Seleção e adaptação da prótese auditiva para o idoso. In: Almeida K, lório MCM. Próteses auditivas: fundamentos teóricos e aplicações clínicas. 2. ed. São Paulo: Lovise; 2003. p. 385-410.

25. Ruschel CV, Carvalho CR, Guarinello AC. Reabilitação auditiva em idosos. Rev Soc Bras Fonoaudiol. 2007; 12(2):95-8.

26. Biz J, Stroher N. Avaliação do benefício do uso de aparelho de amplificação sonora individual (AASI) em idosos. [monografia]. Itajaí (SC): Universidade do Vale de Itajaí; 2007.

27. Costa KCF, Russo ICP, Friedman S. O sentido da deficiência auditiva e do uso de aparelhos de amplificação sonora para idosos. Rev Dist Comun. 2007; 19(3):375-87.

28. Silman S, lório MCM, Mizhahi MM, Parra VM. Próteses auditivas: um estudo sobre seu benefício na qualidade de vida de indivíduos portadores de perda auditiva neurossensorial. Dist Comun. 2004; 16(2):153-65. 